\title{
Pick and Place Robotic Arm Implementation Using Arduino
}

\author{
Ashly Baby ${ }^{1}$, Chinnu Augustine ${ }^{2,}$ Chinnu Thampi ${ }^{3}$ Maria George ${ }^{4}$, \\ Abhilash A $\mathrm{P}^{5}$,Philip C Jose ${ }^{6}$ \\ Department of Electronics and Communication Engineering HKCET, Pampakuda Ernakulam, India
}

\begin{abstract}
A robotic arm is designed using arduino to pick and place the objects via user commands. It will pick and place an object from source to destination safely. The soft catching gripper used in the arm will not apply any extra pressure on the objects. The robot is controlled using android based smart phones through Bluetooth. Based on the commands given by the user the robot moves accordingly. At the receiver end there are four motors interfaced with the micro controller. Two for the vehicle movement and the remaining two are for arm and gripper movement. Blue control application is used for the controlling of robot.
\end{abstract}

Keywords: Pick and place robotic arm, Blue control app, soft catching gripper.

\section{Introduction}

Robotics deals with the design of robots their manufacture and applications. Robotics gained more importance in the modern era since it require less cost to operate than a human labour to do the same task, also once programmed robot will perform better than an experienced human labour. Now a days industry is turning towards computer based monitoring of tasks mainly due to the need for the increased productivity and delivary of the final products with maximum quality. Due to the inflexibility and generally high cost of hard computerization systems lead to the use of industrial robots. In this paper we are introducing a robotic arm which is capable of picking up and placing the objects. The soft catching gripper used here handle objects safely . An android based smart phone which has blue control application is used for the movement of robot. Thus based on the user commands the robot moves and pick and place the objects. The robotic arm used here is similar to a human arm which is programmed to perform the pick and place functions. The remainder of this paper is organized as follows. The section 2 provides information about the existing works. Section 3 gives details of the proposed system. The experimental set up and results were discussed in the section 4.Section 5 concludes the paper.

\section{Existing Works}

[1]Mohamed et.al. introduced a Pick and place robotic arm controlled by Computer vision. Here the robot picks the object at a specific orientation only. The gripper used here is a mechanical gripper. So it can't handle the object safely. Objects in a specific orientation is only picked up by the robotic arm.[2] Anush et.al. introduced Design and Fabrication of Pick and Place Robot to Be Used in Library. Here the robot pick up the books from library and deliver this to the destination. The robotic arm used here can hande objects in any orientation. RFID tags are used to identify the books. This system is capable of doing this specific task only and it's a line following robot. Each RFID has its own path, and this makes the system more complex.[3] N F Begum et.al. is designed an Autonomous android controlled robot design using wireless energy. Here the system works according to voice commands or speech deliverd by the user and the robotic arm is capable of picking up the objects of any type and in any orientation. RF technology is used so line of sight is a major constrain in communication.[4]T Yoshimi et.al. introducing a system for picking up operation of thin objects by robotic arm with two fingered parallel gripper. Thin objects like paper and plastic cards are picked up by this robotic arm. The objects may slide down due to the use of parallel gripper. This method does not provide safety of the object.

\section{Proposed System}

For cost effectiveness and reducing harm to the objects, we introduced a robotic arm with better wireless communication technology and soft catching gripper. The soft catching gripper used here reduces the extra pressure to be applied while picking the object ,thus the objects can be carried without any damage and human effort can be reduced. The robot is controlled remotely using android based smart phone or tablets, so there is no need of complex hardwires to operate this system. This increases the easiness of user. By the use of low power wireless communication technology, the system become more effective and user friendly. Ashly Baby1, Chinnu Augustine2, Chinnu Thampi3, Maria George4,Abhilash A P5,Philip C Jose6 Department of Electronics and Communication Engineering HKCET, Pampakuda Ernakulam, India 


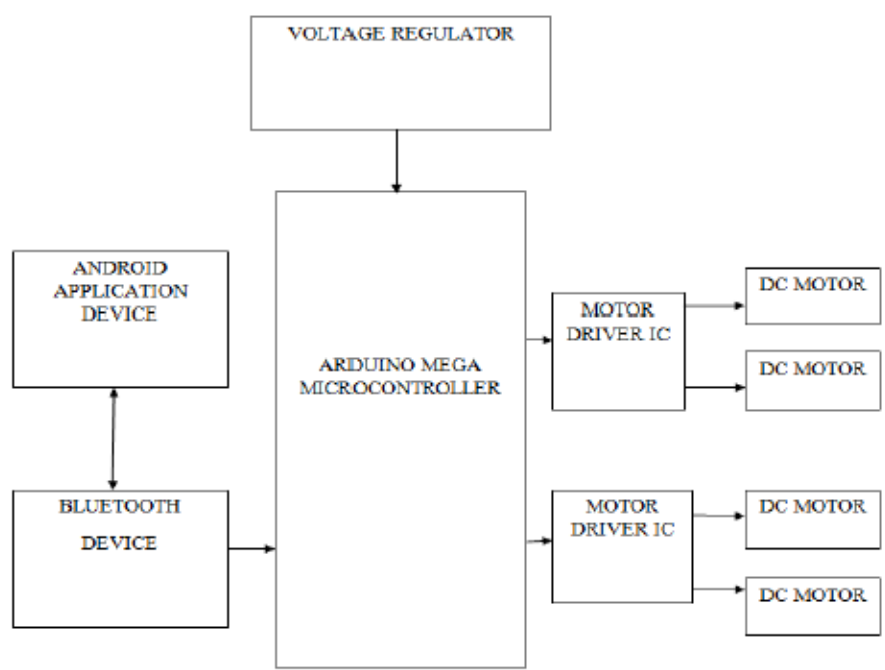

Figure 1 . Block Diagram

Fig 1 shows the proposed system. It mainly comprised of Arduino mega micro controller, bluetooth module(HC- 06), four DC motors with driver IC, voltage regulator. The Bluetooth device, driver IC and voltage regulator are interfaced with the microcontroller. when the user given a command to the microcontroller, it is then checked with the prestored character and if they are same then the robot do the particular operation such as it can move to any direction forward, backward, left, right, arm up, arm down, pick up object and place it .There are four motors are used, two motors are used for the movement of the vehicle and one for the movement of arm and the remaining one for the movement of gripper. The maximum upward and downward movement of arm and closing and opening of jaw is limited by the mechanical push button type switches. For the proper working of motors, driver IC (L293D) are used. Single L293D can handle a set of two DC motors simultaneously. It works on the concept of $\mathrm{H}$-bridge. Voltage regulator provides a regulated voltage for the smooth functioning of the device. Blue control app is used to sending commands to the controller. Blue control is a basic Universal Remote Control for Bluetooth enabled serial devices such as Bluetooth modules connected to a controller. When a button is pressed corresponding ASCII code will send to the controller.

\section{Experimental Setup And Results}

The proposed system is implemented using the Arduino mega 2560 microcontroller. It has 54 input output pins. The operating voltage of mega microcontroller is 5v. The Bluetooth module and four motors are interfaced with the microcontroller. Motor driver L293D receive $12 \mathrm{~V}$ from power supply and drive the motors. Each DC motor is connected to the IN1,IN2 and IN3,IN4 of the driver IC. Movement of the robotic vehicle and arm is based on the direction of rotation of motors. By giving digital high or low values to the motor pins we can rotate it to any direction.

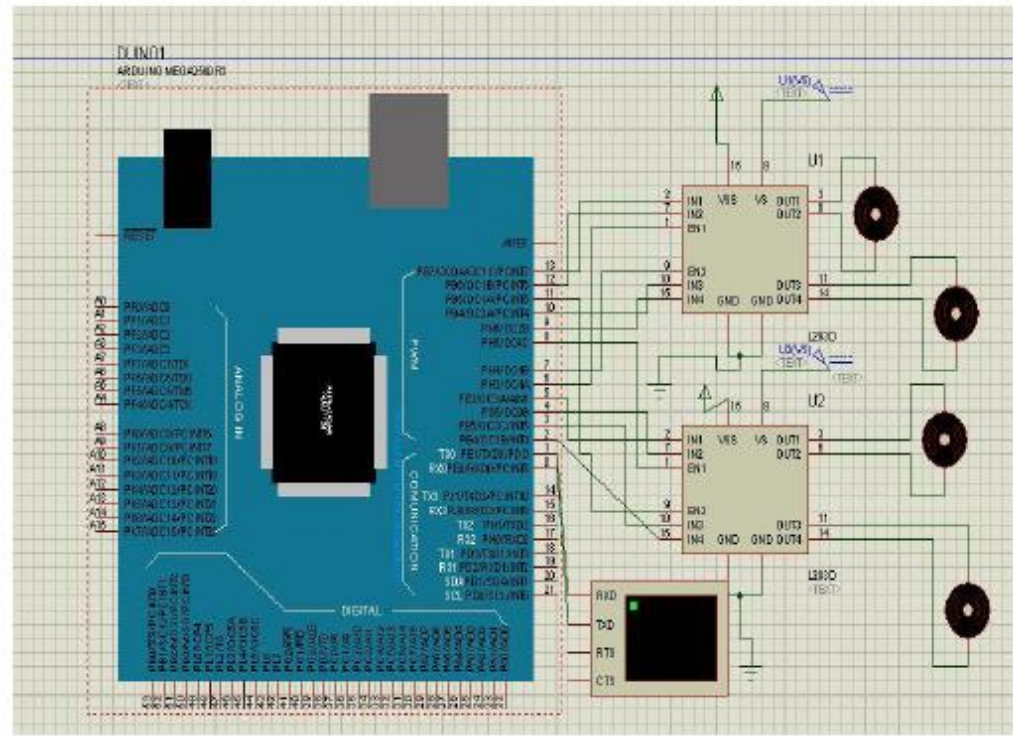

Figure 2 . layout 


\section{FLOW CHART}

\section{Start}
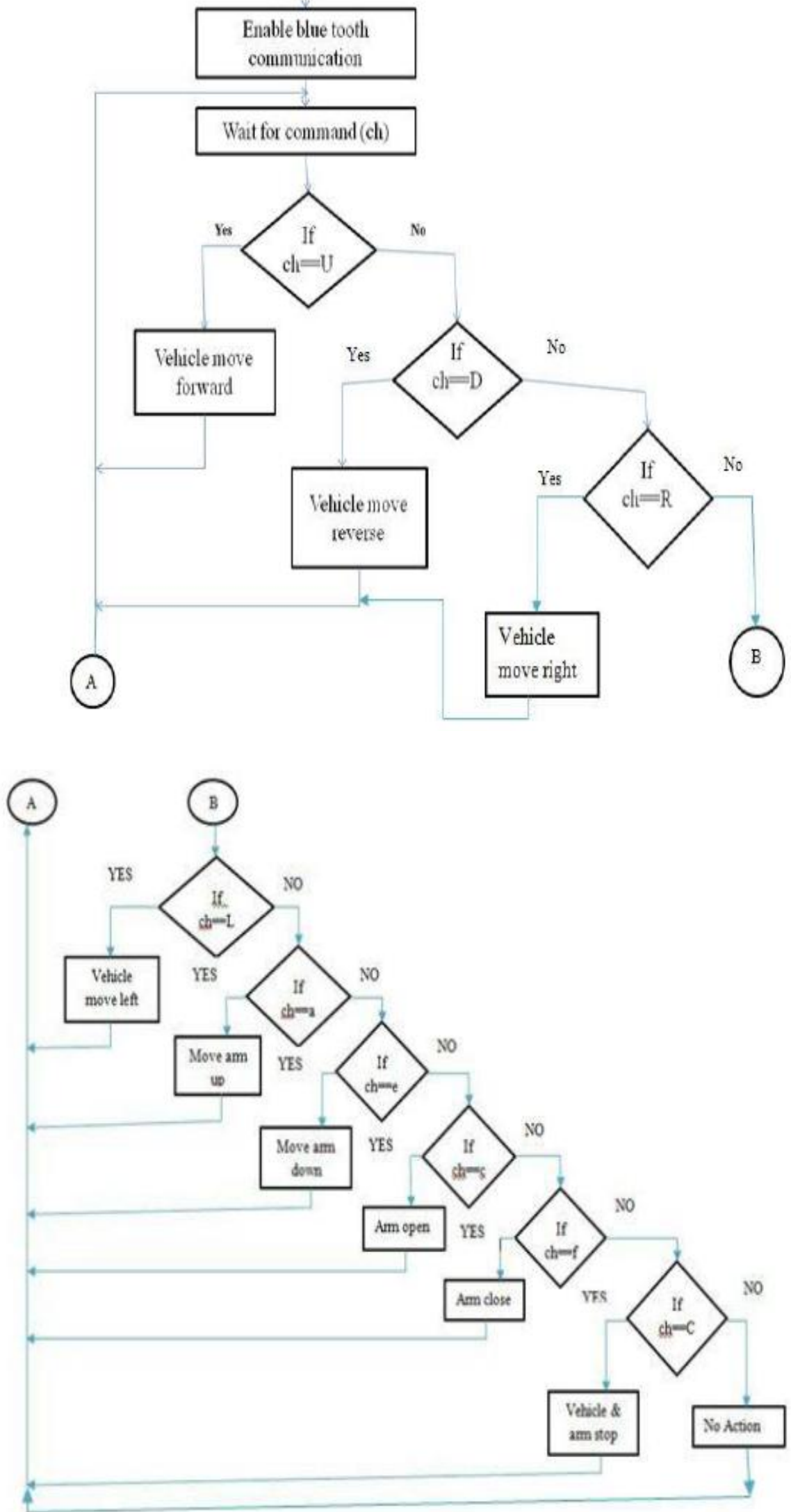

Figure 3.Flow chart 
Figure 3 shows the flow chart of overall working of the system. Bluetooth communication is enabled after power on the system. Once the pairing between the two devices occur, the controller waits for the commands from the user. When the user press a button on the bluecontroll app corresponding ASCII code is send to the controller. The controller checks this with the prestored value, if they are same then corresponding operation done. For example if the user press ' $U$ ' then the operation corresponds to ' $U$ ' is vehicle move forward happens. likewise all other operations are performed. After each command the controller waits for the the next command.

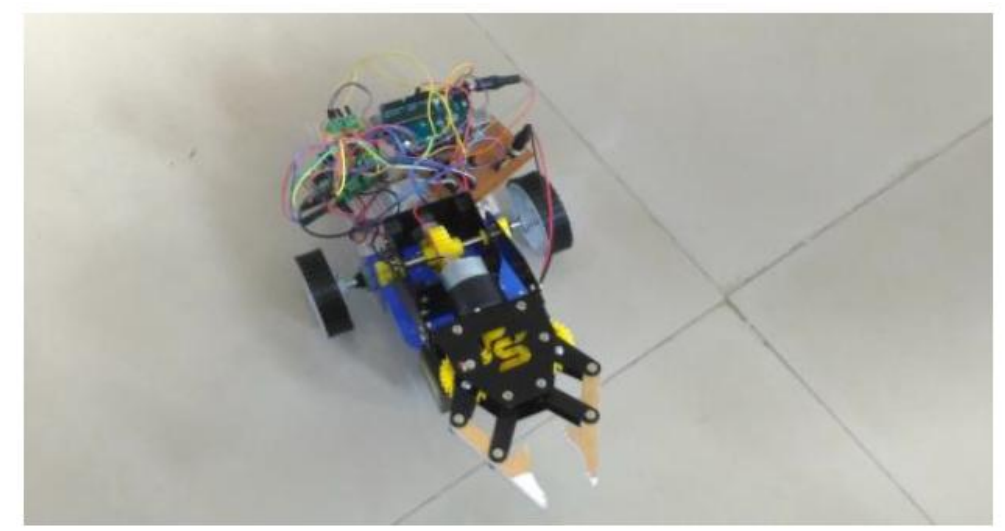

Figure 4.Final Product

Figure 4 shows the hardware setup of final product. The system consist of a vehicle carrying a robotic arm. It will pick and place object from source to destination according to the user command. For that a communicaton is established from user phone to the system through Bluetooth. When the controller receives the command, is compared with the prestored value and do corresponding actions such as, It can move either forward, backward, left or right. In addition to this the arm can be move up or down and the gripper can move away or close for pick and place application.

\section{Conclusion}

A robotic arm is implemented using arduino to pick and place objects more safely without incurring much damage. The robotic arm used here contain a soft catching gripper which safely handle the object. In the modern era time and man power are major constraints for the completion of a task. By the use of our product the industrial activities and hazardous operations can be done easily and safely in a short span of time. The use of soft catching gripper and low power wireless communication technique like Bluetooth makes our system more effective when compared to other systems. The proposed system is capable of lifting only small weights, by introducing high torque providing motor large weights can be picked. A wireless camera can also be implemented to track the movement of the vehicle and thus it can be used in defence purposes. The range is also a limitation it can be enhanced by using a wireless communication technology.

\section{References}

[1]. Mohamed Naufal bin Omar,"Pick and place robotic arm controlled by Computer", Universitite knika IMalaysia, Melaka April 2007.

[2]. Anusha Ronanki , M. Kranthi,"Design and Fabrication of Pick and Place Robot to Be Used in Library”, International Journal of Innovative Research in Science, Engineering and Technology (An ISO 3297: 2007 Certified Organization) Vol. 4, Issue 6, June 2015

[3]. N.F Begum,"Autonomous Android controlled robo design using wireless energy", International Journal of Innovative Research in Advanced Engineering (IJIRAE) ISSN: 2349- 2163 Issue 2, Volume 2 (February 2015)

[4]. Takashi Yoshimi, Naoyuki Iwata, Makoto Mizukawa and Yoshinobu Ando, Member, IEEE," Picking up Operation of Thin Objects by Robot Arm with Two-Fingered Parallel Soft Gripper", Proceedings of the 2012 IEEE International Workshop on Advanced Robotics and its Social Impacts, Technische Universität München, Munich, Germany, May 21 - 23, 2012

[5]. B.O.Omijeh,R.Uhunmwangho,M.Ehikhamenle,"Design Analysis of a Remote Controlled Pick and Place Robotic Vehicle",International Journal of Engineering Research and Development e-ISSN: 2278-067X, p-ISSN: 2278-800X, www.ijerd.com Volume 10, Issue 5 (May 2014), PP.57-68

[6]. John Iovine., "Robots,Androids, and Animations 12 Incredible Projects You Can Build", Second Edition,McGraw- Hill.2002

[7]. Yanjianghuang, ryosukechiba, tamioarai, tsuyoshiueyama and junota. ,"Integrateddesign of multi-robot system for pickand- place tasks",

[8]. Sungwookmoon ,youngjinkim, ho junmyeong, changsookim, namjucha,and dong hwankim . ,"Implementation of smart phone environment

[9]. ATMELATmega48A/PA/88A/PA/168A/PA/328/P [Datasheet] 2 Atmel-8271I-AVR- ATmega-Datasheet10/2014.

[10]. SGS Thomson Microelectronics L293D - L293DD [Datasheet] push-pullfour channel driver with diode .June 1996.

[11]. Fairchild semiconductor KA78XX/KA78XXA 3- Terminal 1A Positive Voltage Regulator Data sheet.2001 Fairchild Semiconductor Corporation. + 